\title{
UM MODELO HIERÁRQUICO PARA PREVISÃO DE PREÇOS DE COMMODITIES AGRÍCOLAS
}

\section{A HIERARCHICAL MODEL TO AGRICULTURAL COMMODITIES PRICES FORECAST}

\author{
Celma de Oliveira Ribeiro* celma@usp.br \\ Anna Andrea Kajdacsy Balla Sosnoski** anna.sosnoski@gmail.com \\ Sydnei Marssal de Oliveira*** sydneim@gmail.com \\ *Universidade de São Paulo \\ ** PETROBRÁS - Petróleo Brasileiro S.A. \\ *** Escola Politécnica - Universidade de São Paulo
}

\begin{abstract}
Resumo: Nos últimos 20 anos tem ocorrido um interesse crescente no comportamento do preço de commodities devido a mudanças no padrão da demanda mundial e o crescimento dos mercados futuros de commodities como instrumento para gestão de portfólios na indústria. A fim de reduzir risco e assegurar preços, os agentes de mercado empregam diferentes estratégias de hedging baseadas no em mercados futuros, sendo imprescindível o uso de modelos de previsão. Neste contexto, o objetivo deste artigo é construir um modelo de previsão para preços à vista de commodities agrícolas com base em um modelo hierárquico. Um primeiro modelo de espaço de estados é ajustado de forma a identificar tendências das séries. Os resultados obtidos são então corrigidos através de redes neurais. Para analisar o comportamento do modelo foram consideradas commodities: soja, álcool e açúcar. Os resultados sugerem que o modelo pode ser uma ferramenta útil para entender os mercados e para previsão de preços de curto prazo.
\end{abstract}

Palavras Chave: Commodities agrícolas. Mercados Futuros. Redes Neurais. Modelos híbridos. Previsão.

Abstract: Last two decades have seen a considerable interest in commodities price behavior as a result of demand pattern changes and the growth of commodities futures markets as a tool to portfolio management in industry. In order to reduce risk and assure prices, agents use different hedging strategies based on futures markets. In this sense forecast models are essential. This paper proposes a mathematical model to agricultural commodity prices forecasting. A hierarchical model is presented. A state space model is considered to identify trends or to describe the structure of the stochastic process. This model is adjusted through a neural network. To analyze the model three commodities were considered: soybeans, alcohol and sugar. The results suggest that this model can be a useful tool to understand markets and to short term forecast.

Key words: Agricultural Commodities., Future market. Neural networks. Hierarchical models. forecast.

\section{INTRODUÇÃO}

O comportamento dos preços de commodities agrícolas tem sido objeto de pesquisa há décadas (PETEE, 1936). A incerteza a respeito dos níveis de produção 
e demanda local e mundial, questões relativas à safra e entressafra e políticas governamentais protecionistas em âmbito mundial estão entre os diversos fatores que tornam o problema de gestão da produção agrícola difícil e desafiante. Em particular, o recente desenvolvimento de substitutos de combustíveis fósseis no mercado mundial, com destaque para o álcool combustível e o biodiesel, tem também levado profissionais do setor e pesquisadores a analisar com maior rigor 0 mercado de commodities agrícolas, com destaque para derivados de cana de açúcar e soja.

Muitas das decisões tomadas pelos produtores, mesmo antes da colheita, presumem o conhecimento de tendências de comportamento de preços em curto e médio prazo. Decisões referentes ao mix de produção, bem como à adoção de políticas de financiamento e construção de estratégias de hedging no mercado de capitais como forma de assegurar preços, são baseadas em expectativas de preços futuros. Também no mercado financeiro é comum empregar o mercado futuro de commodities agrícolas como instrumento para redução de risco de carteiras de investimento. Assim, a previsão do comportamento de preços de commodities é um problema importante e será tratado aqui

Este artigo apresenta um modelo hierárquico para previsão de preços que agrega modelos dinâmicos para séries com tendências e redes neurais, tendo como objetivo seu uso para prever os preços futuros de commodities. Embora com uma estrutura bastante ampla, que permite sua utilização dentro de universo bastante abrangente de problemas, o modelo foi aplicado aos mercados de álcool, soja e açúcar em função da importância das mesmas no mercado futuro nacional e internacional.

O artigo será apresentado como segue. A Seção 2 descreve as abordagens usuais para determinação de preços de commodities agrícolas. Na Seção 3 é apresentado o modelo proposto. Os resultados e conclusões encontram-se nas Seções 4 e 5 respectivamente. 


\section{PREÇOS DE COMMODITIES AGRÍCOLAS}

Ainda que os mercados futuros de commodities agrícolas apresentem liquidez e que o volume de commodities negociadas nos mercados a vista e a termo seja expressivo, peculiaridades destes ativos tornam complexa a elaboração de modelos de apreçamento e gestão de risco. GEMAN (2005) aponta que preços de commodities agrícolas em geral apresentam sazonalidade e possuem alta volatilidade. A autora destaca que a alta volatilidade de preços de commodities se deve a fatores relativos ao clima, deterioração dos produtos agrícolas, armazenamento, entre outros. Além de questões referentes à safra, entressafra e armazenagem, as estratégias de proteção e as barreiras alfandegárias em mercados internacionais e a própria evolução tecnológica são fatores que afetam o comportamento estocástico dos preços e dificultam a sua descrição e previsão.

As transações com commodities podem ser realizadas física - com a entrega do produto - ou financeiramente - através de transações monetárias, sem a entrega do bem - , ao contrário dos mercados de ações ou de títulos, cujas transações são todas realizadas financeiramente (GEMAN, 2005). O risco nestas operações pode ser reduzido através de inúmeras estratégias financeiras. A comercialização direta de mercadorias entre partes - fornecedores e consumidores de matéria prima - em data anterior à entrega da mercadoria é a maneira mais simples e comum de proteção contra oscilações de preço. Inúmeras estratégias podem ser também elaboradas através do uso do mercado de derivativos, construídas através de portfolios de contratos do mercado futuro e de opções (RIBEIRO e WIDONSCK, 2009).

Uma importante abordagem para analisar preços de commodities consiste em descrever a dinâmica dos preços através de processos estocásticos relativamente complexos. Em geral os modelos que descrevem preços a vista empregam processos estocásticos com reversão à média, cujos parâmetros são estimados através de técnicas de otimização. Quando o objetivo é analisar o comportamento de preços de commodities agrícolas no mercado a vista, estes modelos devem incorporar a descrição de fenômenos como o retorno de conveniência (convenience yield) (GEMAN, 2005), uma peculiaridade destes mercados que pode ser entendido como o prêmio financeiro que deve receber o proprietário da commodity física. Os 
modelos variam de acordo com a estrutura empregada para incorporar o retorno de conveniência e com o número de fatores empregados para descrever a incerteza nos preços. Brennan e Schwartz (1985) trabalharam com um modelo de um fator, sendo que modelos com mais de um fator tiveram início com Gibson e Schwartz (1990). Em 1997 Schwartz apresenta um modelo de dois fatores no qual a convenience yield é descrita através de um processo de Orstein-Uhlenbeck com reversão à média, sendo o filtro de Kalman utilizado para determinação dos parâmetros. Posteriormente Schwartz e Smith (2000) utilizaram um modelo de dois fatores que incorporava mudanças de curto e longo prazo nos preços. Sorensen (2002), com base no modelo de Schwartz e Smith introduz a sazonalidade dos preços e também estima os parâmetros do modelo através do filtro de Kalman. Um modelo de três fatores é desenvolvido por Cortazar e Schwartz (2003). Aiube et al. (2008) questionam as hipóteses que possibilitam o uso de filtro de Kalman e apresentam um modelo de filtros de partículas para determinação de preços a vista. O filtro de Kalman é um algoritmo recursivo que permite estimar parâmetros de modelos representados na forma de espaço de estados.

Elliot e Hyndman (2007) descrevem o preço a vista de commodities através de um processo estocástico com reversão à média. A partir de sua representação em espaços de estados, os autores constroem um algoritmo para estimar preços a vista com base em preços futuros.

No caso específico da previsão de preços futuros de commodities é possível empregar modelos de espaços de estado sem que seja necessário considerar o retorno de conveniência. Assim, é possível utilizar abordagens tradicionais de séries temporais. Particularmente quando se pretende construir estratégias de hedging é necessário estimar preços futuros.

\section{MODELOS DE SÉRIES TEMPORAIS}

Há uma extensa literatura a respeito de modelos de previsão de séries temporais, tendo a área apresentado considerável progresso nas últimas décadas (GOOIJER e HYNDMAN, 2006). Modelos adaptativos bastante conhecidos, como os modelos de suavização exponencial são empregados com sucesso em diferentes áreas de conhecimento (GARDNER, 2006). Mais recentemente as redes neurais, 
modelos adaptativos bastante gerais, cujos parâmetros são reajustados à medida que novos dados são apresentados ao modelo, tornaram-se também uma importante ferramenta para previsão. Além de serem modelos não lineares e bastante flexíveis, as redes neurais podem aproximar qualquer função (ZHANG, 2003).

\subsection{Modelos de espaço de estado}

Os modelos de espaço de estado possuem uma estrutura bastante ampla, e generalizam diferentes modelos de previsão. Sua estrutura, na sua forma mais simples, é a seguinte:

$$
\begin{aligned}
& X_{t+1}=A_{t} X_{t}+\varepsilon_{t} \\
& Y_{t}=C_{t} X_{t}+\xi_{t} \quad t \in\{1,2, \ldots N\}
\end{aligned}
$$

onde $Y_{t}$ é um vetor de observações, $X_{t}$ é um processo estocástico (não necessariamente observável), $A_{t}$ é a matriz de transição de estados, $C_{t}$ é a matriz do sistema, e os vetores $\varepsilon_{t}$ e $\xi_{t}$ são ruídos aleatórios não correlacionados. Uma expressiva quantidade de modelos de séries temporais pode ser colocada dentro desta estrutura, sendo que todo modelo linear de séries multidimensionais podem ser assim representado (MORETTIN e TOLOI, 2004). Um modelo específico e bastante conhecido pode ser colocado dentro deste contexto: o modelo de média móvel.

O modelo de médias móveis prevê o próximo valor de uma série com base nos dados históricos. O valor previsto é obtido através da expressão

$$
\frac{X_{t}+X_{t-1}+\ldots+X_{t-r+1}}{r}
$$

onde r é o intervalo de observações para trás que irá se utilizar (lag da média móvel) e $X_{t}$ é o valor da observação realizada no instante t. (MORETTIN e TOLOI, 2004). É imediato que este modelo se escreve como: 


$$
\begin{aligned}
& \Phi_{t+1}=\left[\begin{array}{c}
X_{t} \\
X_{t-1} \\
\vdots \\
X_{t-r+2}
\end{array}\right]=\left[\begin{array}{cccccc}
1 / r & 1 & 0 & & & 0 \\
0 & 1 / r & 1 & & & 0 \\
\vdots & & & \ddots & & \\
0 & 0 & & & 1 / r & 1 \\
0 & 0 & & 0 & 1 / r
\end{array}\right]\left[\begin{array}{c}
X_{t-1} \\
X_{t-2} \\
X_{t-r+1}
\end{array}\right]+\left[\begin{array}{c}
\mathcal{E}_{t} \\
0 \\
\vdots \\
0
\end{array}\right] \\
& Y_{t}=\Phi_{t} t \in\{1,2, \ldots N\}
\end{aligned}
$$

De maneira análoga, o modelo de suavização exponencial realiza uma média ponderada entre os dados históricos e as previsões realizadas anteriormente. As previsões são determinadas a partir da expressão:

$$
X_{t}^{*}=\alpha \cdot X_{t}+(1-\alpha) \cdot X_{t-1}^{*}
$$

onde $X_{t}^{*}$ é o valor da previsão para o período t, $X_{t}$ é o valor da observação obtida na data t e a é a constante de suavização. Gardner (2006) discute detalhadamente este tipo de modelo e apresenta modelos de espaço de estados, que equivalem aos modelos de suavização exponencial.

\subsection{Redes neurais}

As redes neurais são modelos não lineares que consistem de uma estrutura subdividida em camadas, cada uma das quais composta de neurônios. Os neurônios são conectados entre si e pesos determinam a intensidade destas conexões. Estes pesos, os parâmetros do modelo, são obtidos através de técnicas de otimização que minimizam alguma medida de erro.

Um modelo importante é a rede neural feedforward. A primeira camada recebe os dados de entrada. A saída desta camada é entrada da segunda; a saída da segunda é entrada da terceira e assim por diante. Cada neurônio de uma camada recebe informação da camada precedente. A informação é ponderada pelos pesos associados àquela ligação entre os neurônios, e uma função de ativação transforma o resultado em um número - a saída do neurônio. Este processo é repetido através de todas as camadas e a saída da última camada é comparada com as observações, sendo os pesos ajustados de forma a minimizar alguma medida de erro. 
Dentre as vantagens de modelos de rede neurais estão a possibilidade de empregar variáveis qualitativas e não lineares.

\subsection{Modelos híbridos}

Modelos combinados, onde dois ou mais modelos são agregados de forma a aumentar a capacidade preditiva são conhecidos (ZOU ET AL.,2007). Neste caso é possível utilizar modelos com diferentes características estruturais, por exemplo, integrando modelos que consideram não linearidades para descrever a evolução dos preços, com modelos que empregam variáveis qualitativas. Ainda que estes modelos, na maioria das vezes, não forneçam previsões ótimas, em decorrência da hierarquização, são modelos que apresentam bastante flexibilidade. ZHANG (2003) apresenta um modelo que agrega modelos ARIMA e redes neurais, aplicável a séries temporais univariadas.

O modelo aqui proposto consiste numa abordagem hierárquica que integra dois modelos. Para um preço $P_{t}$ considera-se uma decomposição em fatores: $P_{t}=K_{t}+N_{t}$, onde $K_{t}$ é a componente que descreve tendências ou a estrutura de um processo estocástico e $N_{t}$ uma componente não linear descrita através da rede neural. O modelo determina os parâmetros associados a $K_{t}$ e à rede neural de maneira hierarquizada, adotando, portanto uma estratégia sub-ótima. A saída do primeiro modelo é empregada como entrada de um modelo de rede neural, juntamente com outras variáveis exógenas, cuja escolha baseia-se na expertise dos agentes de mercado e nas especificidades do mercado analisado. Um conjunto de variáveis de entrada define uma primeira previsão de preços e a saída deste modelo é empregada como entrada de um modelo de rede neural que refina a qualidade das previsões.

Note que esta estrutura é apropriada tanto para modelos de preços à vista quanto para preços futuros, uma vez que as redes neurais, dentro desta composição, adaptam-se às peculiaridades de cada situação. Os preços futuros de commodities negociados em bolsa refletem as expectativas de oferta e demanda mundiais enquanto que preços a vista dependem muitas vezes peculiaridades de mercados locais nos quais as negociações são realizadas (DOOLEY e LENIHAN, 
2005). Neste contexto, é razoável empregar modelos de híbridos para descrever preços a vista através do filtro de Kalman e corrigi-los através da rede, com o uso de variáveis referentes ao mercado local no qual as commodities são negociadas. De maneira similar é possível empregar modelos de séries temporais para identificar tendências e incorporar informações sobre mercados internacionais na determinação de preços futuros.

\section{RESULTADOS}

Para analisar o desempenho dos modelos híbridos, quatro commodities soja, café arábica, açúcar e etanol - foram consideradas por suas características de liquidez e participação na produção agrícola brasileira. De acordo com o ministério da agricultura, os três principais produtos agrícolas de exportação em 2006, foram exatamente a soja e seus derivados, os produtos da cana, açúcar e etanol, e em terceiro lugar o café. Os dados são apresentados na Tabela 1. Optou-se por analisar os preços à vista, presumindo que o modelo seja empregado por um agente que possui a commodity. O problema de previsão de preços é particularmente importante quando este agente é um produtor agrícola cuja estratégia de produção depende fortemente da variação dos preços. Este é o caso, por exemplo, da produção de usinas sucro alcooleiras em que o usineiro deve decidir qual o mix de produção, à luz de sua expectativa de preços.

Tabela 1: Principais produtos agrícolas exportados

\begin{tabular}{|l|c|c|}
\hline \multirow{2}{*}{$\begin{array}{l}\text { Produtos de } \\
\text { Exportação }\end{array}$} & \multicolumn{2}{|c|}{ 2006 } \\
\cline { 2 - 3 } Soja e derivados & US Milhôes & Particip. \% \\
\hline Açúcar e Alcool & 6844 & $36 \%$ \\
\hline Café & 2666 & $28 \%$ \\
\hline
\end{tabular}

Fonte: Ministério da Agricultura (2006)

Como entrada do primeiro modelo - que descreve tendências - foram utilizadas 96 observações de dados mensais de preços a vista do açúcar, do etanol, do café arábica e da soja, de Janeiro de 2002 a Dezembro de 2009, obtidos no Centro de Estudos Avançados em Economia Aplicada (CEPEA/Esalq). 
A média móvel e a suavização exponencial têm sido utilizadas há décadas pelos agentes dos mercados financeiros tanto para realizar estimativas, por exemplo, para prever a volatilidade de preços de ativos, como dentro de uma abordagem denominada análise técnica, onde estas duas ferramentas são empregadas para determinar momentos de compra e venda de ativos (GARDNER, 2006; COLBY E MEYERS, 1988). Neste artigo estes dois casos particulares são empregados para identificação de tendências, não só para diminuição do esforço computacional, uma vez que reduzem a complexidade do problema, mas também em função de sua popularidade. Para empregar a média móvel é preciso determinar o lag, enquanto que a suavização exponencial depende da constante de suavização $\boldsymbol{\alpha}$, que alisa a série de dados e indica a importância de observações recentes.

Foram determinados parâmetros ótimos para o lag da média móvel e para o parâmetro de suavização, através da minimização do erro quadrático. Os valores ótimos do lag e da constante de suavização $\alpha$ para as quatro commodities são apresentados nas tabelas de resultados. Destaca-se que embora este estudo tenha sido orientado sob a ótica de um produtor, o que levou à opção por modelos simples para descrição de tendências, o modelo proposto pode incorporar trivialmente modelos ARIMA, por exemplo, ou mais gerais, desde que se considere um modelo de espaço de estados, generalizando o trabalho de ZHANG (2003).

Para analisar o preço à vista da soja, do café arábica, do açúcar e do etanol considerou-se como variável de entrada para a rede neural a saída do modelo de previsão (Média Móvel Simples ou Suavização Exponencial) do respectivo produto agrícola. Além desta variável utilizaram-se ainda o como variáveis de entrada 0 preço à vista do petróleo e a taxa de câmbio do dólar. A literatura recente destaca a relação entre o preço de petróleo no mercado mundial e o preço de commodities (CHEN, KUO e CHEN, 2010). Do ponto de vista da produção de commodities o preço do petróleo afeta o preço de commodities de várias maneiras, por exemplo, aumentando custos de produção e de transporte. Em termos de impacto na demanda, algumas commodities competem com produtos derivados do petróleo, ou mesmo por sua capacidade de substituí-lo como fonte de energia (BAFFES, 2007). Assim, adotou-se o preço do petróleo como variável de entrada para a rede neural. A taxa de câmbio foi utilizada uma vez que as transações comerciais em mercados 
agrícolas mundiais, em especial nas bolsas de commodities internacionais, são realizadas em sua maioria nesta moeda.

Para o açúcar e o etanol duas variáveis adicionais foram consideradas: o número de unidades vendidas de veículos bicombustíveis e o preço à vista do etanol, no caso da previsão do açúcar, e o preço à vista do açúcar, no caso da previsão do etanol. Estas duas commodities possuem uma forte relação por serem produzidas com base na mesma matéria prima, a cana de açúcar. Já a produção de veículos pode possui relação com a demanda dos dois produtos citados.

Os preços à vista do petróleo (WTI - West Texas Intermediate) foram obtidos na bolsa de mercadorias de Nova York (NYMEX). A taxa de câmbio foi obtida através do site do Banco Central do Brasil. Dados sobre os veículos bi-combustível, de Janeiro de 2002 a Dezembro de 2009, foram obtidos na Associação Nacional de Veículos Automotivos (ANFAVEA).

A seleção da topologia da rede, da função de transferência e o processo de treinamento adotado seguiu a abordagem de CO e BOOSARAWONGSE (2007). Adotou-se uma rede neural feedforward, com três camadas: uma camada de entrada, uma intermediária e uma camada de saída. A função sigmoidal logarítmica resultou em melhores estimativas e foi escolhida como função de transferência. Os procedimentos de treinamento empregaram 50 observações, tendo-se empregado o algoritmo de Levenberg-Marquardt para treinamento da rede. Várias topologias de rede foram utilizadasm e as que apresentaram o melhor desempenho em termos de treinamento e validação são mostradas nas Figuras 1, 2 e 3.

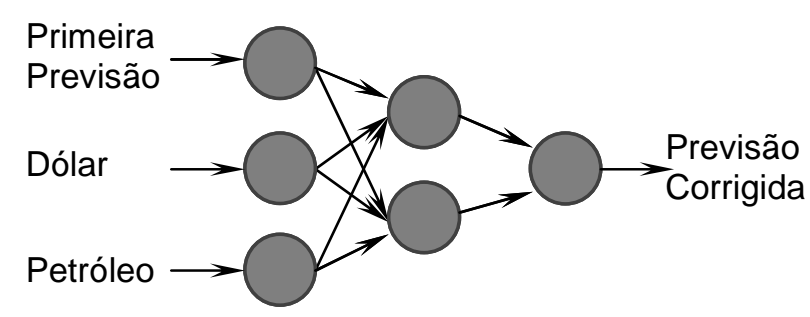

Figura 1: Melhor solução para Soja e Café Arábica

Fonte: Autores. 


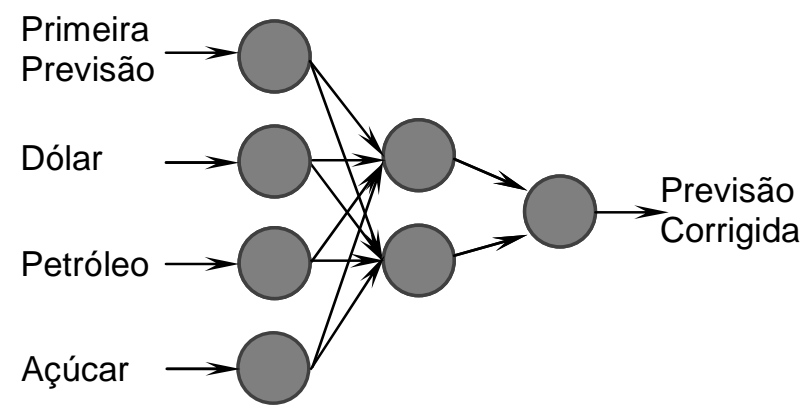

Figura 2: Melhor solução para o Etanol

Fonte: Autores.

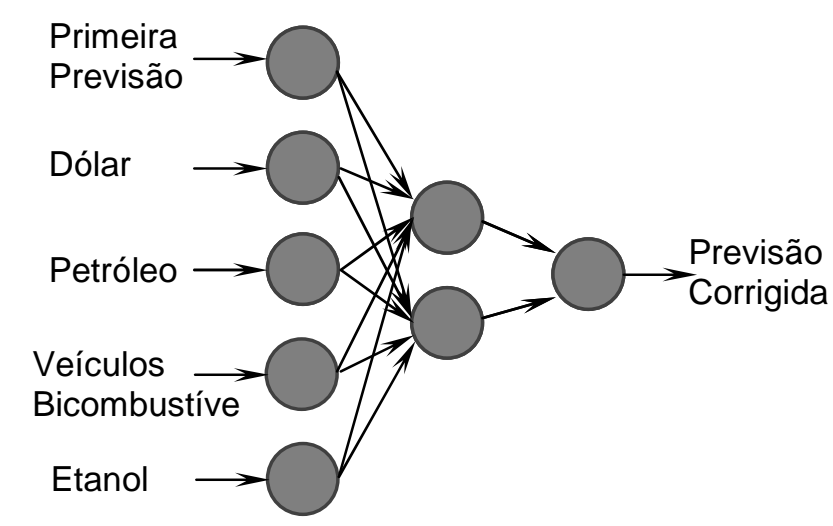

Figura 3: Melhor solução para o Açúcar Fonte: Autores.

Para comparar os modelos, com suavização exponencial e média móvel como entrada, diversas medidas de desempenho foram analisadas, entre elas, o erro médio absoluto (MAE), o erro quadrático médio (MSE), o erro percentual absoluto médio (MAPE) e, por fim, a variância do erro. As expressões de cada uma destas medidas são apresentadas a seguir.

$$
\begin{gathered}
M A E=\frac{1}{n} \sum_{i=1}^{n}\left|e_{i}\right|, \\
M S E=\frac{1}{n} \sum_{i=1}^{n} e_{i}^{2}, \\
M A P E=\frac{1}{n} \sum_{i=1}^{n}\left|\frac{e_{i}}{A_{i}}\right|,
\end{gathered}
$$




$$
\text { Variância }=\frac{1}{n} \sum_{i=1}^{n}\left(e_{i}-\bar{e}\right)^{2},
$$

Nas expressões acima, $\left|\boldsymbol{e}_{\boldsymbol{i}}\right|$ é o erro absoluto no tempo $\boldsymbol{i}$, ou seja, a diferença entre o valor previsto e o valor real. O valor real é $\boldsymbol{A}_{i}$ e $\bar{e}$ é o erro médio.

As medidas de desempenho geradas pelo modelo de médias móveis e pelo modelo de suavização exponencial, assim como o lag e a constante de suavização $\alpha$ são apresentados nas tabelas 2 e 3 , respectivamente.

Tabela 2: Medidas de Desempenho para Médias Móveis

\begin{tabular}{|l|c|c|c|c|c|c|c|c|c|c|}
\hline \multirow{2}{*}{ Produtos } & Variáveis & \multicolumn{5}{|c|}{ Médias Móveis } & \multicolumn{4}{c|}{ Redes Neurais } \\
\cline { 3 - 11 } & Exógenas & lag & MAE & Var & MSE & MAPE & MAE & Var & MSE & MAPE \\
\hline Açúcar & DPVE & 2 & 0.61 & 21.24 & 21.39 & $22.56 \%$ & 0.01 & 18.04 & 17.85 & $9.74 \%$ \\
\hline Etanol & DPA & 2 & 0.85 & 121.33 & 120.79 & $18.92 \%$ & 1.18 & 78.40 & 78.98 & $8.81 \%$ \\
\hline Café & DP & 2 & 2.58 & 420.21 & 422.48 & $38.31 \%$ & 0.73 & 385.22 & 381.74 & $6.67 \%$ \\
\hline Soja & DP & 2 & 0.33 & 11.33 & 11.32 & $22.97 \%$ & 0.09 & 9.91 & 9.81 & $6.63 \%$ \\
\hline
\end{tabular}

Fonte: Autores.

Tabela 3: Medidas de Desempenho para Suavização Exponencial

\begin{tabular}{|l|c|c|c|c|c|c|c|c|c|c|}
\hline \multirow{2}{*}{ Produtos } & Variáveis & \multicolumn{4}{|c|}{ Suavização Exponencial } & \multicolumn{4}{c|}{ Redes Neurais } \\
\cline { 3 - 11 } & Exógenas & $\alpha$ & MAE & Var & MSE & MAPE & MAE & Var & MSE & MAPE \\
\hline Açúcar & DPVE & 1.000 & 0.42 & 15.13 & 15.15 & $8.15 \%$ & 0.19 & 14.40 & 14.29 & $9.52 \%$ \\
\hline Etanol & DPA & 0.976 & 0.62 & 90.08 & 89.53 & $8.72 \%$ & 1.75 & 68.45 & 70.79 & $7.83 \%$ \\
\hline Café & DP & 0.904 & 1.87 & 336.43 & 336.44 & $6.13 \%$ & 1.70 & 313.93 & 313.55 & $5.75 \%$ \\
\hline Soja & DP & 0.947 & 0.20 & 9.82 & 9.76 & $5.97 \%$ & 0.02 & 7.69 & 7.61 & $5.77 \%$ \\
\hline
\end{tabular}

Fonte: Autores.

A coluna referente às variáveis exógenas identifica as variáveis utilizadas na rede neural através de letras, sendo que $D$ representa a taxa de câmbio do dólar, $P$ o preço do petróleo, $\mathrm{V}$ o número de veículos bicombustível produzidos, $\mathrm{E}$ o preço à vista do etanol e A o preço a vista do açúcar.

O modelo de Suavização Exponencial forneceu resultados melhores que os obtidos através do modelo de Médias Móveis. Consequentemente a redução dos erros decorrente do uso da rede neural não foi tão expressiva. Esse foi o caso do erro percentual absoluto médio (MAPE) para o açúcar e para o etanol, onde não houve melhoria. Com exceção do MAPE para o açúcar e etanol, em todos os outros 
casos o modelo por Redes Neurais promoveu acentuada redução em todas as medidas de desempenho.

No caso da soja e do café o modelo por suavização exponencial seguido do modelo por redes neurais mostrou um desempenho muito significativo.

\section{CONCLUSÕES}

Este artigo apresentou um modelo para previsão de preços de commodities agrícolas que combina as abordagens tradicionais baseadas em séries temporais com modelos de redes neurais. O modelo permite que as abordagens que analisam a dinâmica de evolução de preços - através da descrição de processos estocásticos que modelam fenômenos como o retorno de conveniência - sejam empregadas em conjunto com modelos mais gerais. As redes neurais permitem que o primeiro modelo seja corrigido, através do emprego uso de variáveis que afetem especificamente a commodity sendo analisada.

Os resultados obtidos sugerem que os modelos híbridos propostos são úteis para prever preços de commodities e para identificar a dependência destes preços em relação a variáveis exógenas que estejam associadas a características micro ou macroeconômicas da commodity. A inclusão de variáveis exógenas, através das redes neurais, se mostrou efetiva na melhoria do desempenho do modelo de previsão de tendências. Informações sobre oferta e demanda mundiais de cada uma das commodities analisadas poderiam eventualmente melhorar ainda mais o desempenho do modelo, porém essa informação não estava disponível no horizonte de tempo considerado.

Em termos das variáveis empregadas na previsão, o modelo proposto confirma o resultado de CHEN, KUO e CHEN, (2010), que indica que o preço do petróleo afeta o preço de commodities, assim como o taxa de câmbio, confirmando a expectativa de correlação, uma vez que todas as culturas avaliadas são os principais produtos agrícolas de exportação brasileiros.

O modelo hierárquico com redes neurais permite que variáveis exógenas sejam consideradas na previsão de preços sem que se assuma uma forma estrutural a priori. Com isso, o tomador de decisão pode empregar variáveis (qualitativas ou quantitativas) que considere relevantes na formação do preço. Assim, o modelo é 
bastante abrangente, sendo aplicável até mesmo a situações em que o preço depende fortemente de variáveis regionais.

Há duas extensões naturais deste trabalho. O primeiro refere-se ao uso do filtro de Kalman como modelo em substituição ao modelo de suavização exponencial, uma vez que o filtro é capaz de acomodar modelos clássicos para a descrição de processos estocásticos que descrevem a evolução de preços de commodities Outras extensões menores do trabalho incluem o estudo de diferentes variáveis relacionadas associadas às commodities analisadas e também estender o uso do modelo a outras commodities.

\section{REFERÊNCIAS}

AIUBE, F.A.L., BAIDYA T.K.N., TITO E.A.H. Analysis of commodity prices with the particle filter, Energy Economics, v. 30, n.2, p. 597-605, 2008.

BAFFES, j., Oil spills on other commodities, Resources Policy, v. 32, p. 126 -134, 2007.

BRENNAN, M. J.; SCHWARTZ, E. S. Evaluating natural resource investments. The Journal of Business. v.58, n.2, p.135-157, abr. 1985.

CHEN, S., KUO, H., CHEN, C. Modeling the relationship between the oil price and global food prices, Applied Energy, v. 87, p. 2517-2525, 2010.

CO, H. C.; BOOSARAWONGSE, R. Forecasting thailand's rice export: statistical techniques vs. artificial neural networks, Computers \& Industrial Engineering, v. 53, p. 610-627, 2007.

COLBY, R.W.; MEYERS, T. A. The encyclopedia of technical market indicators, Down Jones-Irwin, 1988.

CORTAZAR, G.; SCHWARTZ, E.S. Implementing a stochastic model for oil futures prices, Energy Economics, v. 25, p. 215-238, 2003.

DOOLEY, G., LENIHAN, H. An assessment of time series methods in metal price forecasting" Resources Policy, v. 30, n. 3, p. 208-217, 2005.

ELLIOT, R. J. e HYNDMAN C.B. Parameter estimation in commodity markets: a filtering approach, Journal of Economic Dynamics and Control, v. 31, n.7, $p$ .2350-2373, 2007.

GARDNER JR E.S. Exponential smoothing: the state of the art - Part II", International Journal of Forecasting, v. 22, n.4, p. 637-666, 2006. 
GEMAN, H. Commodities and commodity derivatives: modeling and pricing for agriculturals, metals and energy. Wiley, 2005.

GIBSON, R.; SCHWARTZ, E. S. Stochastic convenience yield and the pricing of oil contingent claims. The Journal of Finance. v.45, n.3, p.959-976, jul., 1990.

GOOIJER,J.G.; HYNDMAN, R.J. 25 years of time series forecast, International Journal of Forecasting, v. 22, p 443-473, 2006.

MINISTÉRIO DA AGRICULTURA - SECRETARIA DE POLÍTICA AGRÍCOLA, Exportações agropecuárias - produtos selecionados US\$ FOB, 2006.

Disponível em: <http://www.agricultura.gov.br/>. Acesso em: 22 jun. 2010.

MORETTIN, P.A.; TOLOI, C.M.C. Análise de séries temporais. 1. ed. São Paulo: Blücher, 2004.

PETEE, E.W., Long Term Commodity Price Forecasting - 1850 to 1930." The journal of Business of the University of Chicago, v. 9, n. 2, p. 95, 1936.

RIBEIRO, C. O.; WIDONSCK, C. A. Agronegócio no Brasil: uma perspectiva financeira. In: SAVOIA, J. R. F. Saint Paul Editora, 2009. Cap. 5.

SCHWARTZ, E. S. Equilibrium forward curves for commodities. The Journal of Finance, v.52, n.3, p.923-973, jul. 1997.

SCHWARTZ, E. S.; SMITH, J. E. Valuing long-term commodity assets. Management Science. v.46, n.7, p.893-911, Jul. 2000.

SORENSEN C., Modeling Seasonality in Agricultural Commodity Futures", The Journal of Future Markets, p. 393-430, 2002.

ZHANG, G. P., Time Series Forecasting Using a Hybrid ARIMA and Neural Network Model., Neurocomputing, v. 50, p. $159-175,2003$.

ZOU, H.F.; XIA, G.P.; YANG, F.T.; WANG H.Y. An investigation and comparison of artificial neural networks and time series models for Chinese food grain prices, Neurocomputing, v. 70, p. 2913-2923, 2007.

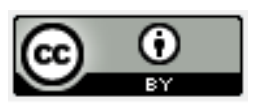

Artigo recebido em 25/03/2009 e aceito para publicação em 03/10/2010. 